
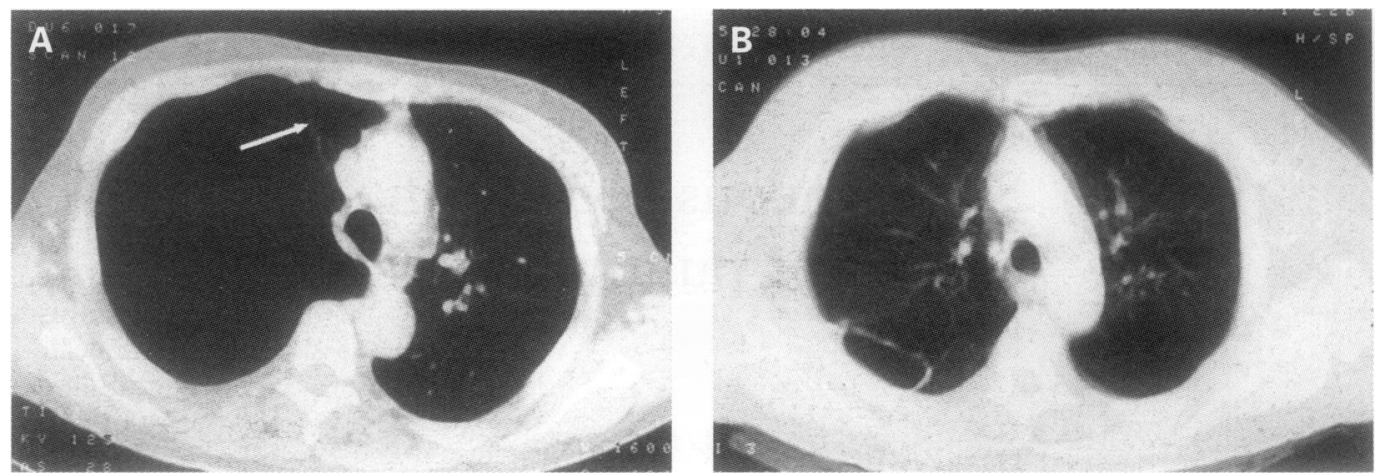

Computed tomographic scan of the chest from (A) 1991 showing a giant bulla in the right lung with significant shift of the mediastinum to the left and $(B)$ in 1993 showing a dramatic reduction in the size of the bulla. Note that the lung is almost completely re-expanded and the mediastinal shift is resolved.

inflammation may further obstruct already compromised bronchial communications with the bullae resulting in a closed space. Eventually fluid and then air resorption leads to regression of the bulla. If this mechanism is valid, one might speculate that other known causes of airway obstruction such as tumour, mucous plugging, or blood clot could also lead to shrinkage of the bulla. A retrospective review from Japan reported three cases of lung bulla regression associated with bronchogenic cancer, although no details on individual cases were provided. ${ }^{10}$

Our case is of interest not only because of the rarity with which spontaneous regression has been reported in the literature, but also because it was associated with such dramatic improvements in the radiological picture and pulmonary function. Unlike earlier reports, this occurred in the absence of overt infection or tumour. Re-expansion of compressed lung and reversal of mediastinal shift was accompanied by improved pulmonary blood flow. Substantial increases in $\mathrm{FEV}_{1}$, as documented in this patient, are sometimes seen following surgical bullectomy in well selected patients. Our patient was not thought to be a good candidate for bullectomy because of diffuse emphysema, which underlines the difficulty in selecting patients for surgery.

The Chief, Bureau of Medicine and Surgery, Navy Department, Washington, DC, Clinical Investigation Program sponsored this report \#84-16-1968-495, as required by HSETCINST $6000.41 \mathrm{~A}$. The views expressed in this article are those of the authors and do not reflect the official policy or position of the Department of the Navy, Department of Defense, or the United States Government.

1 Thurlbeck WM. Chronic airflow obstruction. In: Pathology of the lung. Stuttgart, New York: Thieme Medical Publishers, 1988:549-50.

2 Boushy SF, Kohen R, Billig DM, Heiman MJ. Bullous emphysema: clinical, roentgenologic and physiologic study of 49 patients. Dis Chest 1968;54:327-34.

3 Stone DJ, Schwartz A, Feltman JA. Bullous emphysema. A long-term study of the natural history and the effects of therapy. Am Rev Respir Dis 1960;94:493-507.

4 CIBA Guest Symposium. Terminology, definitions, and classification of chronic pulmonary emphysema and related conditions. Thorax 1959;14:286-99.

5 Murphy DM, Fishman AP. Bullous disease of the lung. In: Fishman AP, ed. Pulmonary diseases and disorders. New York: McGraw-Hill, 1988:1219-22.

6 Morgan MD, Edwards CW, Morris J, Matthews HR. Origin and behaviour of emphysematous bullae. Thorax 1989; and beha

7 Rubin EH, Buchberg AS. Capricious behavior of pulmonary bullae developing fluid. Dis Chest 1968;54:546-9.

8 Rothstein E. Infected emphysematous bullae. Report of five cases. Am Rev Tuberc 1954;69:287-96.

9 Douglas AC, Grant IW. Spontaneous closure of large pulmonary bullae. A report on three cases. $B r$ Tuberc Dis Chest 1958;33:335-8.

10 Tsutsui M, Araki Y, Shirakusa T, Inutsuka S. Characteristic radiographic features of pulmonary carcinoma associated with large bulla. Ann Thorac Surg 1988;46:679-83.
Cardiothoracic

Centre,

Liverpool L14 3PE,

UK

G E Wilson

C C Evans

Correspondence to:

Dr C C Evans.

Received 9 September 1994

Returned to authors

30 November 1994

Revised version received

20 February 1995

Accepted for publication

24 February 1995

\section{Sternocostoclavicular hyperostosis presenting with thoracic sinus formation}

\author{
G E Wilson, C C Evans
}

\author{
Abstract \\ Sternocostoclavicular hyperostosis (SCCH) \\ is a condition which is well described in the \\ Japanese literature but is rare in Western \\ Europe. It is characterised by pain and
}

A 75 year old woman of Ashkenazi Jewish extraction was referred because of increasing shortness of breath. For five years she had suffered from recurrent clavicular problems. Initially this had been swelling, pain, and stiffness around the medial ends of the clavicles 


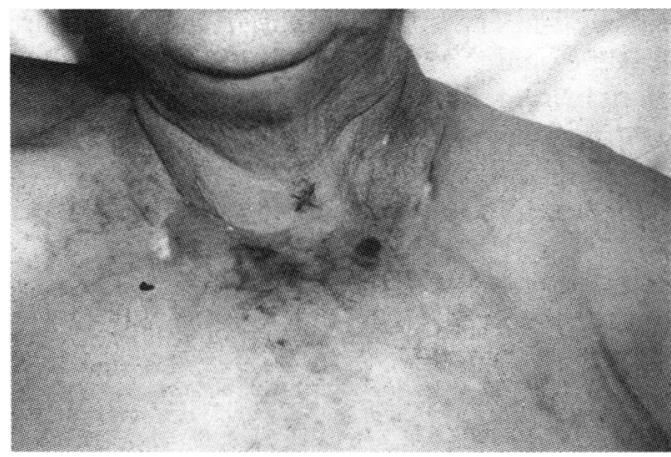

Figure 1 Discharging sinuses over the upper chest wall and right venous congestion.

that had progressed to chronic discharging sinuses on the upper anterior chest wall. She had developed pustular psoriasis affecting the soles of the feet and the palms of the hands.

On examination she weighed $67 \mathrm{~kg}$ and was $145 \mathrm{~cm}$ tall. She was not clubbed or cyanosed. The jugular venous pressure on the right side was elevated and non-pulsatile. The veins over the right breast and upper arm were engorged On the left side of the neck there were discharging sinuses with deformity of the area (fig 1). The right side had a large fluctuant mass at the head of the sternoclavicular joint and a pocketed firmer mass over the right sternomastoid. A surgical scar from her hemicolectomy for diverticulosis still had a dressing on it two and a half years following surgery.

Investigations at the clinic were as follows: ECG revealed sinus rhythm with evidence of right atrial enlargement (' $P$ ' pulmonale). Lung function tests showed a mixed and severe obstructive and restrictive pattern with forced expiratory volume in one second $\left(\mathrm{FEV}_{1}\right) 0.571$ (36\%), forced vital capacity (FVC) $0.91(45 \%)$, and $\mathrm{FEV}_{1} \%$ 64. Chest radiography showed apparent thickening of the anterior end of the left clavicle with some bone destruction (fig 2) The right clavicle was not clearly seen but a possible opacity was noted in the underlying lung.

Ten $\mathrm{ml}$ of pus was aspirated from one of the lesions and sent for urgent staining. This failed to reveal the presence of any acid and alcohol fast bacilli (AAFB) and subsequent culture was negative. Her full blood count revealed

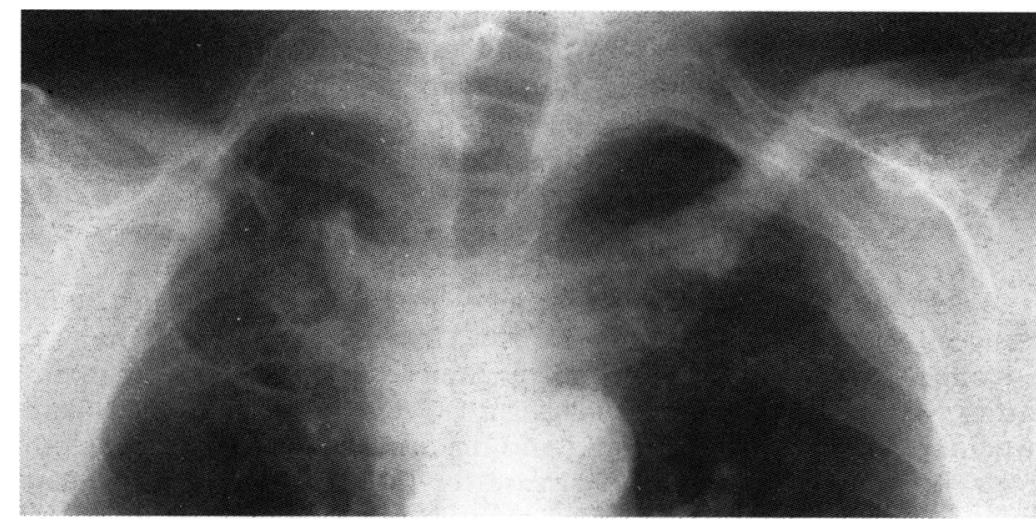

Figure 2 Chest radiograph showing bony destruction of clavicles. haemoglobin of $10.9 \mathrm{~g} / \mathrm{dl}$, white cell count of $7 \cdot 2 \times 10^{9} / 1$ (lymphocytes $26 \cdot 2 \%$, monocytes $10 \cdot 3 \%$, granulocytes $63 \cdot 5 \%$ ). Her erythrocyte sedimentation rate (ESR) was $46 \mathrm{~mm}$ in the first hour. Serum calcium was normal and the alkaline phosphatase was raised at $171 \mathrm{U} / 1$ (normal 10-110). Rheumatoid factor and antinuclear factor were negative. The sinuses were swabbed repeatedly and specimens were sent for microbiological examination and culture. The swabs failed to grow any organisms including bacteria, fungi, AAFB, or actinomyces. A computed tomographic (CT) scan showed bilateral destruction of the clavicles and confirmed the presence of a mass in the right upper chest which extended to the right lobe of the thyroid gland. This appeared to be compressing the superior vena cava but not the trachea. In view of the sterile discharging sinuses, antituberculous treatment with rifampicin and isoniazid was started. After eight weeks of treatment there had been no resolution of symptoms nor of the rate of discharge from the sinuses and the medication was therefore stopped. Nebulised salbutamol, $5 \mathrm{mg}$ four hourly, was given for her airways obstruction with an improvement in her spirometric parameters. A fibreoptic bronchoscopic examination was carried out which showed a normal endobronchial tree. Two years later her physical signs remain unchanged.

\section{Discussion}

Sternocostoclavicular hyperostosis ( $\mathrm{SCCH})$ is a condition with an equal sex distribution whose peak incidence occurs in middle age. The original reports were from Japan in 1967, although since then cases have been described in America and Europe (there does not appear to be an increased incidence in Japanese migrants to these areas). The disorder was first reported by Sasaki ${ }^{1}$ who described a case of bilateral hyperostosis of the clavicles associated with pustulosis palmaris and plantaris. No single aetiological agent has been defined and culture of bone biopsy specimens or resected material has failed to demonstrate an infective cause. $^{2}$ There is debate over the pathogenesis of the disease. Kholer $e t a l^{3}$ proposed that it is an ossifying periostitis which begins within bone and progresses to a generalised hyperostosis, but Fritz et $a l^{4}$ have argued that it is primarily a rheumatological rather than an orthopaedic condition. The diagnosis is primarily clinical and radiological. The most frequently abnormal laboratory investigations include increased ESR, C-reactive protein and alkaline phosphatase. Radionuclide studies show uptake in the sternoclavicular region and may show extrasternal involvement.

Radiological features of SCCH are said to be characteristic and have been classified into three stages by Resnick. ${ }^{5}$ In the case described here the changes corresponded with stage II, with marked destruction of the sternoclavicular joints, the clavicle, and formation of an ossific mass. Extrinsic compression of the subclavian vein and brachial plexus neuropathies due to the effect of local pressure have been reported 
and may require decompressive surgery. ${ }^{67} \mathrm{CT}$ scanning or MRI may reveal the characteristic proliferation of restrosternal tissue, but a biopsy specimen may be required to exclude malignancy. A biopsy specimen was not taken in this case due to the overall poor health of the patient.

As far as we are aware, there are no previous reports of sinus formation occurring in $\mathrm{SCCH}$, either over the affected area or peripherally.

The differential diagnosis of $\mathrm{SCCH}$ includes malignancies such as plasmacytoma involving the sternum and metastatic thyroid or oral cavity carcinomas. Non-malignant causes include diffuse idiopathic skeletal hyperostosis, Paget's disease condensing osteitis, and osteomyelitis.

Treatment is usually conservative as no specific therapy has been shown to alter the course of the disease. Following stage II, which may last for many years, the final stage III is characterised by ankylosis of the affected joints and ossification of the cartilage of the ribs. Limited symptomatic relief may be gained with the use of non-steroidal anti-inflammatory agents and corticosteroids.

The authors wish to thank Mr A Jones, Dr J Verbov, Dr H Carty.

1 Sasaki T. A case with osteomyelitis of the bilateral clavicles as in Seikeigeka 1967;2:333-7 (in Japanese).

2 Chigira $M$, Maehara $S$, Nagase $M$, Ogimi $T$, Udagawa $E$. Sternoclavicular hyperostosis. A report of nineteen cases with special reference to etiology and treatment. $\mathcal{F}$ Bon oint Surg $[\mathrm{Am}]$ 1986;68:103-12.

3 Kholer H, Uehlinger E, Kutzner J, et al. Sternocostoclavicular hyperostosis: painful swelling of the sternum, clavicles and upper ribs. Report of two new cases. Ann Intern Med 1977; 87:192-4.

4 Fritz P, Baldauf G, Wilke HJ, Reitter I. Sternocostoclavicular hyperostosis: its progression and radiological features. A study of 12 cases. Ann Rheum Dis 1992;51:658-64.

5 Resnick D. Sternocostoclavicular hyperostosis. AfR 1980; 135:1278-80.

6 Jirik FR, Stein HB, Chalmers A. Clavicular hyperostosis with enthesopathy, hypergammaglobulinaemia and thoracic outenthesopathy, hypergammaglobulinaemia and thor.

7 Haenel LC, Bradway WR, Constantini PJ. Thrombophlebitis complicating sternocostoclavicular hyperostosis. Postgrad Med $\mathcal{F} 1980 ; 68: 113-8$

\section{Lymphangitis carcinomatosa complicating primary malignant peritoneal mesothelioma}

Paul S Craft, Martin S Reading, Sanjiv Jain, Ross A O’Neil

\begin{abstract}
A patient with malignant peritoneal mesothelioma and a diffuse pulmonary infiltrate is described. Computed tomographic scanning suggested lymphangitis carcinomatosa. This was confirmed on transbronchial biopsy to be due to metastatic mesothelioma.

(Thorax 1996;51:552-553)
\end{abstract}

Keywords: peritoneal mesothelioma, lymphangitis carcinomatosa, metastasis.

Department

$S$ Jain

Radiology Department R A O'Neil

Woden Valley Hospital, Canberra, ACT 2605, Australia

Correspondence to: Dr P S Craft.

Received 5 January 1995 Accepted for publication 4 April 1995 an annual incidence of between 0.7 and per million. ${ }^{12}$ Increasing incidence has been reported in many countries including the United States and, in particular, Australia. ${ }^{13}$ The disease is strongly linked to asbestos exposure, particularly crocidolite. Approximately $10 \%$ of mesotheliomas arise from the peri- toneum. ${ }^{4}$ Lymphangitis carcinomatosa is characterised histologically by diffuse permeation of tumour cells within pulmonary lymphatics. We are not aware of any previously reported examples of lymphangitis carcinomatosa due to malignant mesothelioma.

\section{Case report}

A 52 year old man presented with a two month history of weight loss and upper abdominal pain. There was a history of asbestos exposure 20 years earlier. A chest radiograph, taken at the onset of symptoms, was normal. An abdominal computed tomographic (CT) scan showed a diffuse omental mass. Needle biopsy yielded cytologically abnormal mesothelial cells consistent with mesothelioma.

One month later the patient developed a non-productive cough and exertional dyspnoea. Auscultation of the lung fields revealed bilateral fine basal crackles. The chest radiograph on this occasion showed a widespread reticulonodular pattern with septal thickening. A high resolution CT scan of the thorax revealed diffuse nodular thickening with polygon formation (fig 1). Bronchoscopic examination was normal. Transbronchial biopsy specimens showed abnormal epithelioid malignant cells with mild nuclear pleomorphism and prominent nucleoli. These cells were identical to those obtained from the previous omental needle biopsy. Ultrastructurally they showed long branching microvilli consistent with mesothelioma (fig 2). Cytotoxic chemotherapy with cisplatin and doxorubicin was associated with stable disease for three months. The patient died eight months after presentation from respiratory failure due to progressive disease. 